\title{
Association between lactate clearance during post-resuscitation care and neurologic outcome in cardiac arrest survivors treated with targeted temperature management
}

Jung Chang Kim ${ }^{1}$, Byung Kook Lee', Dong Hun Lee', Yong Hun Jung', Yong Soo Cho', Sung Min Lee', Seung Joon Lee², Chi Ho Park², Kyung Woon Jeung ${ }^{1}$

'Department of Emergency Medicine, Chonnam National University Hospital, Gwangju, Korea

${ }^{2}$ Department of Emergency Medicine, Seonam University Myongji Hospital, Goyang, Korea

Objective We investigated the association between lactate clearance or serum lactate levels and neurologic outcomes or in-hospital mortality in cardiac arrest survivors who were treated with targeted temperature management (TM).

Methods A retrospective analysis of data from cardiac arrest survivors treated with TM between 2012 and 2015 was conducted. Serum lactate levels were measured on admission and at 12,24 , and 48 hours following admission. Lactate clearance at 12, 24, and 48 hours was also calculated. The primary outcome was neurologic outcome at discharge. The secondary outcome was in-hospital mortality.

Results The study included 282 patients; 184 (65.2\%) were discharged with a poor neurologic outcome, and 62 (22.0\%) died. Higher serum lactate levels at 12 hours (odds ratio [OR], 1.157; $95 \%$ confidence interval [Cl], 1.006 to 1.331), 24 hours $(\mathrm{OR}, 1.320 ; 95 \% \mathrm{Cl}, 1.084$ to 1.607), and 48 hours (OR, 2.474; 95\% Cl, 1.459 to 4.195$)$ after admission were associated with a poor neurologic outcome. Furthermore, a higher serum lactate level at 48 hours $(\mathrm{OR}, 1.459 ; 95 \% \mathrm{Cl}, 1.181$ to 1.803) following admission was associated with in-hospital mortality. Lactate clearance was not associated with neurologic outcome or in-hospital mortality at any time point after adjusting for confounders.

Conclusion Increased serum lactate levels after admission are associated with a poor neurologic outcome at discharge and in-hospital mortality in cardiac arrest survivors treated with TM. Conversely, lactate clearance is not a robust surrogate marker of neurologic outcome or in-hospital mortality.

Keywords Heart arrest; Lactic acid; Prognosis; Induced hypothermia
elSSN: 2383-4625

Received: 24 October 2016 Revised: 30 December 2016 Accepted: 4 January 2017

Correspondence to: Byung Kook Lee Department of Emergency Medicine, Chonnam National University Hospital, 42 Jebong-ro, Dong-gu, Gwangju 61469, Korea

E-mail: bbukkuk@hanmail.net

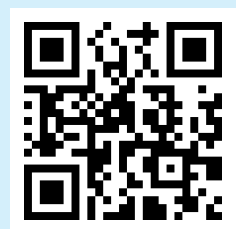

How to cite this article:

Kim JC, Lee BK, Lee DH, Jung YH, Cho YS, Lee SM, Lee SJ, Park CH, Jeung KW. Association between lactate clearance during post-resuscitation care and neurologic outcome in cardiac arrest survivors treated with targeted temperature management. Clin Exp Emerg Med 2017;4(1):10-18.

This is an Open Access article distributed under the terms of the Creative Commons Attribution Non-Commercial License (http:// creativecommons.org/licenses/by-nc/4.0/). 


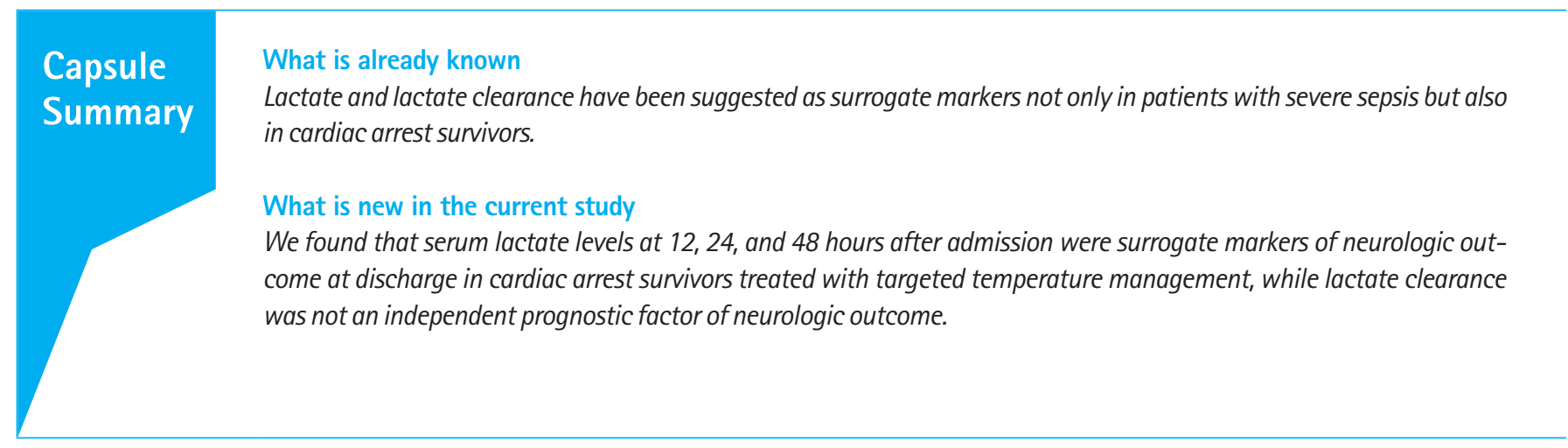

\section{INTRODUCTION}

Advances in post-cardiac arrest care involving targeted temperature management (TM) and goal-directed therapy has improved clinical outcomes, and, thus, post-cardiac arrest care is recommended as a standard therapy in cardiac arrest survivors. ${ }^{1-6} \mathrm{Al}-$ though a target blood pressure has not been fully elucidated, postresuscitation care includes hemodynamic goals to optimize tissue perfusion in addition to TTM. ${ }^{5,6}$ Lactate is a metabolite from anaerobic glycolysis and is an indicator of inadequate tissue perfusion. Serum lactate has been used as a surrogate marker of mortality in patients with severe sepsis independent of organ failure. 'Serum lactate has also been useful as a surrogate marker in cardiac arrest survivors during the TM and non-TTM era. ${ }^{7-14}$ Elevated serum lactate has been associated with increased mortality or a poor neurologic outcome. ${ }^{7-14}$ Furthermore, lactate clearance from serial measurements of serum lactate was suggested as another surrogate marker in cardiac arrest survivors. ${ }^{11-13} \mathrm{~A}$ decrease in lactate clearance has been associated with increased mortality or a poor neurologic outcome. ${ }^{11-13}$ Therefore, serial measurements of serum lactate are recommended for hemodynamic management after the return of spontaneous circulation (ROSC) in cardiac arrest survivors. ${ }^{6,15}$

There are several studies in the literature exploring the association between serum lactate or lactate clearance and clinical outcome in cardiac arrest survivors. ${ }^{714}$ Previous studies have analyzed serum lactate levels obtained 24 hours after admission but, have used a relatively small sample size.,10-12 We hypothesized that higher serum lactate levels and a lower lactate clearance rate during early post-resuscitation care are associated with increased in-hospital mortality or a poor neurologic outcome. We aimed to examine the difference in lactate clearance between neurologic outcome groups and in-hospital mortality groups. Furthermore, we evaluated the association between serum lactate levels on admission and 12,24, and 48 hours after admission and neurologic status at discharge or in-hospital mortality.

\section{METHODS}

\section{Study design and population}

We performed a retrospective observational cohort study including comatose cardiac arrest survivors treated with TTM at Chonnam National University Hospital, Gwangju, Korea between January 2012 and December 2015. This study was approved by the institutional review board of Chonnam National University Hospital.

Cardiac arrest survivors over 16 years-of-age who had completed TTM were included in the present study. Patients were excluded based on the following criteria: death during TM; transfer during $\Pi \mathrm{TM}$; receipt of the $\Pi \mathrm{TM}$ protocol with a different target temperature $\left(<32^{\circ} \mathrm{C}\right.$ or $\left.>34^{\circ} \mathrm{C}\right)$ or hypothermia duration (48 or 72 hours); extracorporeal membrane oxygenation applied during post-cardiac arrest care; and incomplete lactate data.

\section{TTM protocol}

TTM was induced with ice packs, intravenous cold saline, and cooling devices including feedback-controlled endovascular catheters (COOLGARD3000 Thermal Regulation System, Alsius Corporation, Irvine, CA, USA) or surface cooling devices (Blanketrol II, Cincinnati Subzero Products, Cincinnati, OH, USA; Artic Sun Energy Transfer Pads, Medivance Corp., Louisville, KY, USA). Core temperature was monitored using an esophageal temperature probe. Remifentanil and midazolam were used for sedation and analgesia. During the 24-hour maintenance phase, a target temperature of $33 \pm 1^{\circ} \mathrm{C}$ was maintained. Upon completion of the maintenance phase, patients were rewarmed at a target rate of $0.25^{\circ} \mathrm{C} / \mathrm{hr}$. Advanced critical care such as oxygenation, ventilation, glucose control, and hemodynamic optimization were provided according to the guidelines. Serum lactate levels were obtained on admission and at 12, 24, and 48 hours after admission.

\section{Data collection and outcomes}

The following data were obtained for each patient: age, sex, comorbidities, first monitored rhythm, etiology of cardiac arrest, loca- 
tion of cardiac arrest (out-of-hospital or in-hospital), presence of a witness on collapse, bystander cardiopulmonary resuscitation, downtime, Glasgow Coma Scale (GCS) score after ROSC, epinephrine dose used during the intra-arrest period, glucose levels after ROSC, initial temperature, pre-induction time, induction duration, rewarming duration, lactate levels (initial and at 12, 24, and 48 hours after admission), lactate clearance (at 12, 24, and 48 hours), vital status at hospital discharge (alive or dead), and neurologic outcome at discharge. The sequential organ failure assessment (SOFA) score within the first 24 hours after admission was used to assess the severity of multiple organ dysfunction. ${ }^{16}$ Neurologic outcome was assessed using the Glasgow-Pittsburgh Cerebral Performance Category (CPC) at discharge and was recorded as CPC 1 (good performance), CPC 2 (moderate disability), CPC 3 (severe disability), CPC 4 (vegetative state), and CPC 5 (brain dead or death). ${ }^{17}$ Neurologic outcome was dichotomized as either good (CPC 1 and 2) or poor (CPC 3 to 5). The primary outcome was neurologic outcome at discharge. The secondary outcome was in-hospital mortality.

\section{Data analysis}

Continuous variables are given as median values with interquartile ranges according to the results of the normality test. MannWhitney U-tests were conducted for comparisons of continuous variables. Categorical variables are presented as frequencies and percentages. Comparisons of categorical variables were performed using chi-square or Fisher exact tests, as appropriate. Logistic regression analysis was used to examine the association between serum lactate levels and neurologic outcome at discharge or in-hospital mortality, after adjusting for confounders. The significant variables in the univariate analyses were included in the multivariate logistic regression model. Backward selection was used to obtain the final model. The goodness-of-fit of the final model was evaluated using the Hosmer-Lemeshow test. A repeated-measures analysis of variance was conducted to assess lactate change over time. Post-hoc analysis at each time point was performed using the pairwise Mann-Whitney U-test with Bonferroni correction. Data were analyzed using PASW Statistics ver. 18 (SPSS Inc., Chicago, IL, USA). A two-sided significance level of 0.05 was used for statistical significance.

\section{RESULTS}

\section{Patient population}

During the study period, 439 cardiac arrest survivors were treated with TM. As summarized in Fig. 1, 38 were treated with extracorporeal membrane oxygenation, 35 died during TM, 47 were treated with a different TM protocol, 7 were transferred, and 30

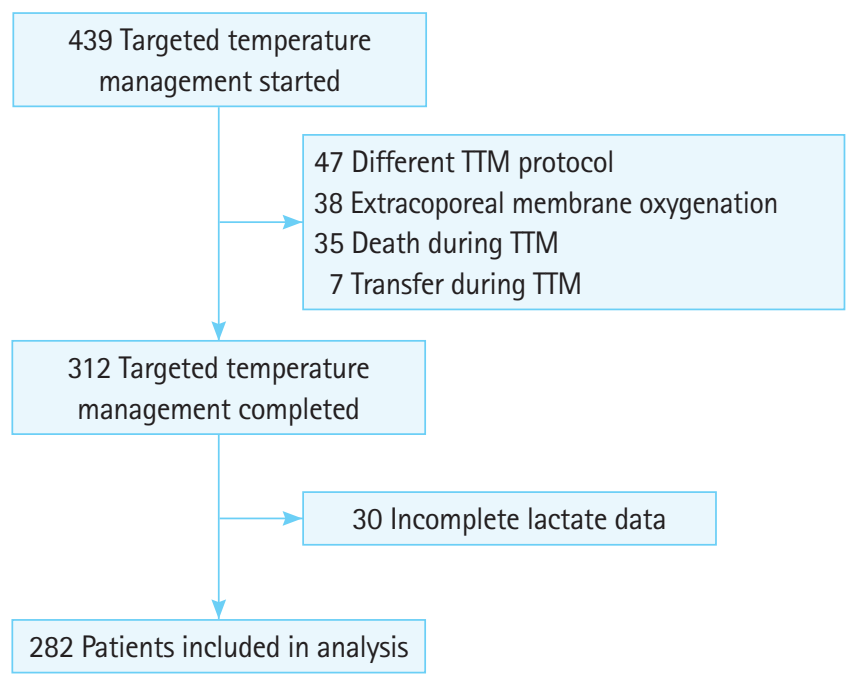

Fig. 1. A schematic diagram showing the selection process of patients for analysis. TMM, targeted temperature management.

had incomplete lactate data. Finally, 282 patients were included in this study (Fig. 1).

Baseline characteristics are shown in Table 1. The median age was 60.5 (interquartile range, 48.8 to 70.3 ) years, 100 patients (35.5\%) had a shockable rhythm, 161 (57.1\%) had a cardiac etiology, 229 (81.2\%) experienced an out-of-hospital cardiac arrest, 207 (73.4\%) were witnessed on collapse, 174 (61.7\%) received bystander cardiopulmonary resuscitation, and the median downtime was 27.0 (interquartile range, 15.0 to 37.0) minutes (Table 1). Poor neurologic outcome at discharge and in-hospital mortality were reported in 184 (65.2\%) and 62 (28.2\%) patients, respectively.

\section{Clinical outcomes}

Table 1 shows the baseline characteristics according to neurologic outcome and in-hospital mortality. Patients with a good neurologic outcome were younger, had a lower incidence of diabetes, were more likely to have a shockable rhythm and cardiac etiology, had a higher incidence of witnessed collapse, required less epinephrine, had a shorter downtime, and had a lower SOFA score, as compared with patients with a poor neurologic outcome.

Survivors were also younger, had a lower incidence of comorbidities (diabetes and renal disease), were more likely to have a shockable rhythm and cardiac etiology, were more likely to have had an out-of-hospital cardiac arrest, required less epinephrine, had a higher GCS score, and had a lower SOFA score, as compared with non-survivors.

Table 2 shows the parameters of TTM according to neurologic outcome and survival. Patients with a good neurologic outcome had a higher initial temperature, shorter pre-induction time, and a longer induction time than patients with a poor neurologic out- 
Table 1. Baseline characteristics of the overall study population and the population stratified by neurologic outcome and survival status

\begin{tabular}{|c|c|c|c|c|c|c|c|}
\hline Characteristics & Total $(n=282)$ & $\begin{array}{l}\text { Good neurologic } \\
\text { outcome }(n=98)\end{array}$ & $\begin{array}{l}\text { Poor neurologic } \\
\text { outcome }(n=184)\end{array}$ & P-value & $\begin{array}{l}\text { Survivor } \\
(n=220)\end{array}$ & $\begin{array}{c}\text { Non-survivor } \\
(n=62)\end{array}$ & P-value \\
\hline Age (yr) & $60.5(48.8-70.3)$ & $54.0(43.8-62.3)$ & $64.0(51.0-72.8)$ & $<0.001$ & $59.0(46.3-69.0)$ & $66.0(51.0-75.3)$ & 0.013 \\
\hline Male sex & $183(64.9)$ & $71(72.4)$ & $112(60.9)$ & 0.052 & $143(65.0)$ & $40(64.5)$ & 0.944 \\
\hline \multicolumn{8}{|l|}{ Comorbidities } \\
\hline CAD & $45(16.0)$ & 19 (19.4) & $26(14.1)$ & 0.251 & $36(16.4)$ & $9(14.5)$ & 0.726 \\
\hline Heart failure & $26(9.2)$ & $8(8.2)$ & $18(9.8)$ & 0.654 & $19(8.6)$ & $7(11.3)$ & 0.523 \\
\hline Hypertension & $114(40.4)$ & $34(34.7)$ & $80(43.5)$ & 0.152 & $86(39.1)$ & $28(45.2)$ & 0.390 \\
\hline Diabetes & $72(25.5)$ & $14(14.3)$ & $58(31.5)$ & 0.002 & $47(21.4)$ & $25(40.3)$ & 0.002 \\
\hline Pulmonary disease & $10(3.5)$ & $1(1.0)$ & $9(4.9)$ & 0.173 & 7 (3.2) & $3(4.8)$ & 0.462 \\
\hline Renal disease & $33(11.7)$ & $8(8.2)$ & $25(13.6)$ & 0.177 & $17(7.7)$ & $16(25.8)$ & $<0.001$ \\
\hline CVA & $14(5.0)$ & $4(4.1)$ & $10(5.4)$ & 0.777 & $13(5.9)$ & $1(1.6)$ & 0.317 \\
\hline Hepatic disease & $6(2.1)$ & $1(1.0)$ & $5(2.7)$ & 0.668 & $4(1.8)$ & $2(3.2)$ & 0.616 \\
\hline First monitored rhythm & & & & $<0.001$ & & & 0.022 \\
\hline VF/pulseless VT & $100(35.5)$ & $62(63.3)$ & $38(20.7)$ & & $87(39.5)$ & $13(21.0)$ & \\
\hline PEA & $68(24.1)$ & $22(22.4)$ & $46(25.0)$ & & $52(23.6)$ & $16(25.8)$ & \\
\hline Asystole & $111(39.4)$ & 13 (13.3) & $98(53.3)$ & & 78 (35.5) & $33(53.2)$ & \\
\hline Unknown & $3(1.1)$ & $1(1.0)$ & $2(1.1)$ & & $3(1.4)$ & $0(0.0)$ & \\
\hline Etiology & & & & $<0.001$ & & & 0.001 \\
\hline Cardiac & $161(57.1)$ & $80(81.6)$ & $81(44.0)$ & & 135 (61.4) & $26(41.9)$ & \\
\hline Other medical & 64 (22.7) & $11(11.2)$ & $53(28.8)$ & & $44(20.0)$ & $20(32.3)$ & \\
\hline Asphyxia & 37 (13.1) & $3(3.1)$ & 34 (18.5) & & $31(14.1)$ & $6(9.7)$ & \\
\hline Drug overdose & $18(6.4)$ & $4(4.1)$ & $14(7.6)$ & & $10(4.5)$ & $8(12.9)$ & \\
\hline Drowning & $2(0.7)$ & $0(0.0)$ & $2(1.1)$ & & $0(0.0)$ & $2(3.2)$ & \\
\hline Location & & & & 0.650 & & & 0.007 \\
\hline Out-of-hospital & 229 (81.2) & $81(82.7)$ & $148(80.4)$ & & $186(84.5)$ & $43(69.4)$ & \\
\hline In hospital & 53 (18.8) & $17(17.3)$ & 36 (19.6) & & 34 (15.5) & $19(30.6)$ & \\
\hline Witnessed & $207(73.4)$ & $81(82.7)$ & $126(68.5)$ & 0.010 & $163(74.1)$ & $44(71.0)$ & 0.623 \\
\hline Bystander CPR & $174(61.7)$ & $64(65.3)$ & $110(59.8)$ & 0.364 & $136(61.8)$ & 38 (61.3) & 0.940 \\
\hline Epinephrine (mg) & $2(1-4)$ & $1(0-3)$ & $3(2-5)$ & $<0.001$ & $2(1-4)$ & $4(2-6)$ & $<0.001$ \\
\hline Downtime (min) & $27.0(15.0-37.0)$ & $21.0(15.0-30.0)$ & $30.0(15.5-40.0)$ & $<0.001$ & $25.0(15.0-35.0)$ & $30.0(15.0-41.8)$ & 0.186 \\
\hline GCS & $3(3-3)$ & $3(3-6)$ & $3(3-3)$ & $<0.001$ & $3(3-4)$ & $3(3-3)$ & 0.004 \\
\hline Glucose (mg/dL) & 227 (168-289) & $216(156-286)$ & 233 (181-293) & 0.122 & 218 (165-289) & $244(188-300)$ & 0.207 \\
\hline SOFA score & $9(7-11)$ & $8(6-10)$ & $9(7-12)$ & $<0.001$ & $8(6-11)$ & $11(9-14)$ & $<0.001$ \\
\hline
\end{tabular}

Values are presented as median (interquartile range) or number (\%).

$C A D$, coronary artery disease; CVA, cerebrovascular accident; VF, ventricular fibrillation; VT, ventricular tachycardia; PEA, pulseless electrical activity; CPR, cardiopulmonary resuscitation; GCS, Glasgow Coma Scale; SOFA, sequential organ failure assessment.

Table 2. Parameters of targeted temperature management of the population stratified by neurologic outcome and survival status

\begin{tabular}{|c|c|c|c|c|c|c|c|}
\hline & Total $(n=282)$ & $\begin{array}{l}\text { Good neurologic } \\
\text { outcome }(n=98)\end{array}$ & $\begin{array}{l}\text { Poor neurologic } \\
\text { outcome }(n=184)\end{array}$ & P-value & $\begin{array}{l}\text { Survivor } \\
(n=220)\end{array}$ & $\begin{array}{c}\text { Non-survivor } \\
\quad(n=62)\end{array}$ & P-value \\
\hline Initial temperature $\left({ }^{\circ} \mathrm{C}\right)$ & $36.0(35.1-36.8)$ & $36.4(35.6-37.0)$ & $35.8(34.8-36.5)$ & $<0.001$ & 36.1 (35.1-36.8) & $35.9(34.8-36.5)$ & 0.260 \\
\hline Pre-induction time (min) & 210 (153-292) & 198 (145-268) & 225 (159-308) & 0.036 & 214 (153-290) & $209(145-311)$ & 0.933 \\
\hline Induction duration (hr) & $2.3(1.3-3.5)$ & $2.9(2.0-4.3)$ & $2.0(1.0-3.0)$ & $<0.001$ & $2.4(1.5-3.7)$ & $1.8(1.0-3.0)$ & 0.031 \\
\hline Rewarming duration (hr) & $12.0(12.0-14.1)$ & $12.0(12.0-13.1)$ & $13.0(12.0-15.0)$ & 0.122 & $12.3(12.0-14.0)$ & $12.0(12.0-16.0)$ & 0.701 \\
\hline
\end{tabular}

Values are presented as median (interquartile range).

come. Survivors had a longer induction time than non-survivors, whereas other TMM characteristics were not different (Table 2).

\section{Serum lactate levels and lactate clearance}

Serum lactate levels differed between neurologic outcome groups and decreased over time $(P<0.001)$ with no interaction between neurologic outcome and time ( $P=0.318)$ (Fig. 2). Post-hoc analysis showed that serum lactate levels differed between neurologic outcome groups at 12, 24, and 48 hours after admission (Fig. 2). Lactate clearance at all time points differed between the good 

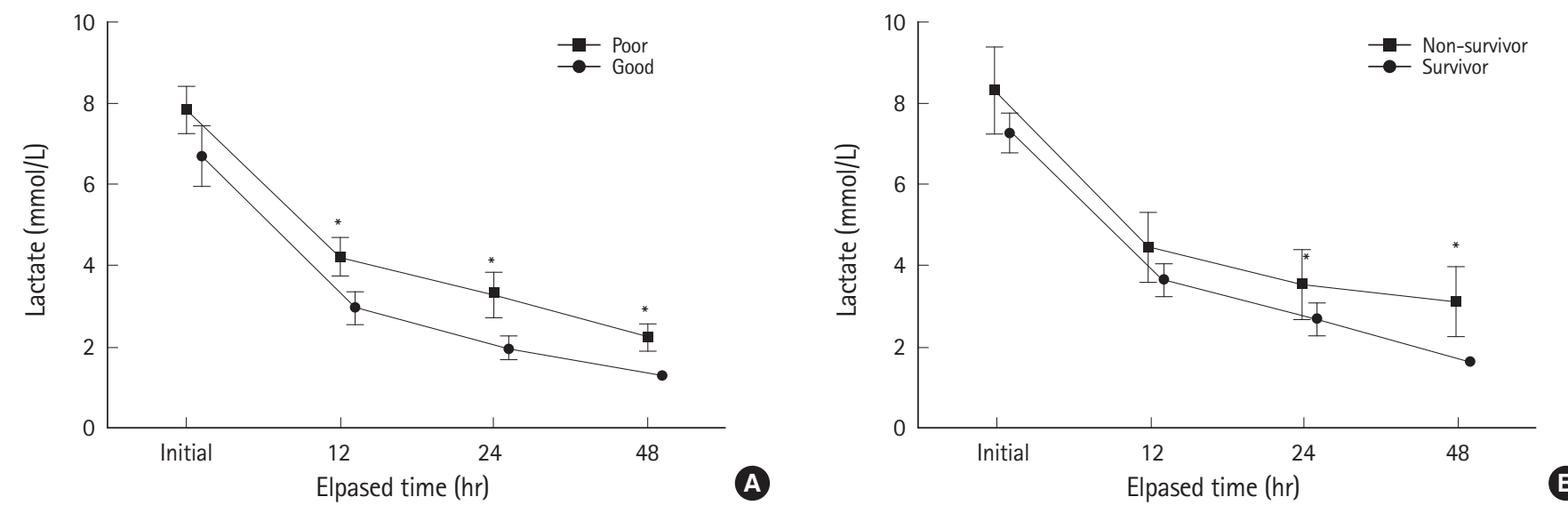

Fig. 2. Comparison of repeatedly measured serum lactate levels between outcome groups. (A) There was no interaction between neurologic outcome and time $(P=0.318)$. Serum lactate levels decreased over time $(P<0.001)$ and differed between the good and poor neurologic outcome groups $(P<0.001)$. Post-hoc analysis adjusted with Bonferroni correction showed that the serum lactate levels at 12, 24, and 48 hours after admission were significantly different between the good and poor neurologic outcome groups. (B) There was no interaction between in-hospital mortality and time ( $P=0.444)$. Serum lactate levels differed between survivors and non-survivors $(P=0.001)$. Post-hoc analysis adjusted with Bonferroni correction showed that the serum lactate level at 48 hours after admission was higher in non-survivors. ${ }^{*} \mathrm{P}<0.05$.
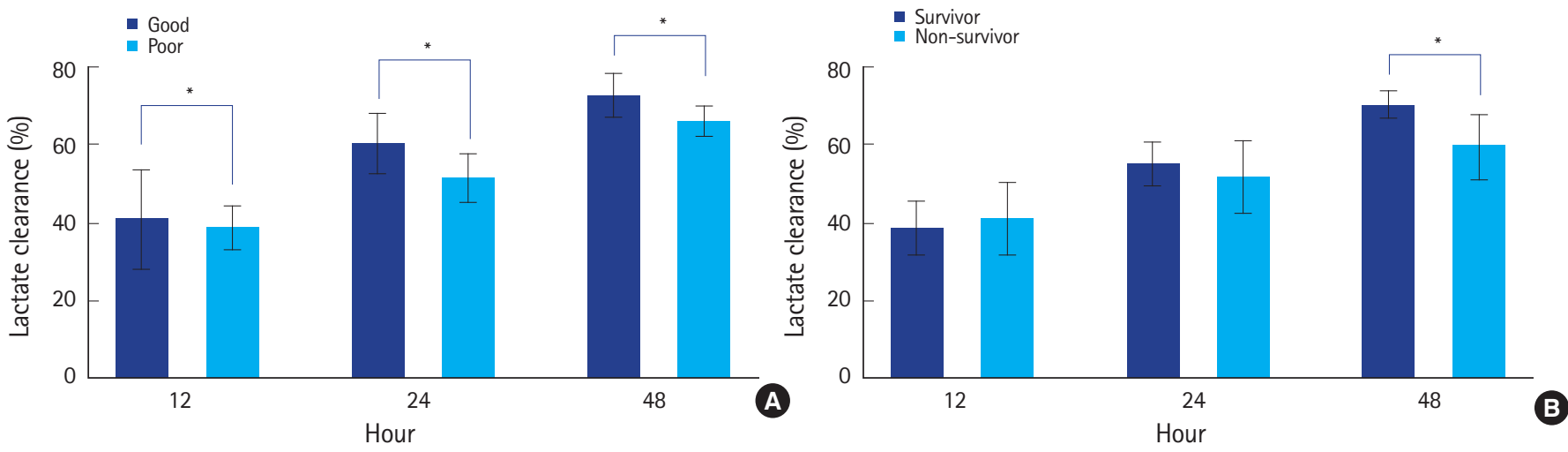

Fig. 3. Comparison of lactate clearance between outcome groups. (A) Lactate clearance at 12, 24, and 48 hours after admission was significantly different between good and poor neurologic outcome groups. (B) Lactate clearance at 12 and 24 hours after admission was not associated with in-hospital mortality. Lactate clearance at 48 hours after admission was significantly lower in non-survivors. ${ }^{*} \mathrm{P}<0.05$.

Table 3. Multivariate logistic regression analysis of characteristics associated with a poor neurologic outcome

\begin{tabular}{lcccc}
\hline & & \multicolumn{3}{c}{ OR (95\% Cl) } \\
\cline { 2 - 5 } Characteristics & Model 1 & Model 2 & Model 3 & Model 4 \\
\hline Age (yr) & $1.038(1.016-1.060)^{*}$ & $1.038(1.016-1.061)^{*}$ & $1.039(1.016-1.062)^{*}$ & $1.037(1.015-1.061)^{*}$ \\
Shockable rhythm & $0.253(0.117-0.549)^{*}$ & $0.240(0.110-0.524)^{*}$ & $0.236(0.106-0.526)^{*}$ & $0.224(0.100-0.504)^{*}$ \\
Cardiac etiology & $0.267(0.119-0.601)^{*}$ & $0.283(0.126-0.637)^{*}$ & $0.273(0.121-0.615)^{*}$ & $0.272(0.119-0.620)^{*}$ \\
Downtime (min) & $1.053(1.029-1.079)^{*}$ & $1.050(1.025-1.075)^{*}$ & $1.048(1.022-1.074)^{*}$ & $1.045(1.020-1.070)^{*}$ \\
Glasgow Coma Scale & $0.581(0.442-0.764)^{*}$ & $0.605(0.461-0.794)^{*}$ & $0.584(0.439-0.776)^{*}$ & $0.581(0.438-0.769)^{*}$ \\
Lactate levels (mmol/L) & & & & NA \\
Initial & $1.049(0.962-1.143)$ & NA & NA & NA \\
At 12 hours & NA & $1.157(1.006-1.331)^{*}$ & NA \\
At 24 hours & NA & NA & $1.320(1.084-1.607)^{*}$ & NA \\
At 48 hours & NA & NA & NA & $2.474(1.459-4.195)^{*}$ \\
\hline
\end{tabular}

Serum lactate levels at the different time points were separated into different multivariate models. Model 1 included initial lactate level, model 2 included lactate level at 12 hours after admission, model 3 included lactate level at 24 hours, and model 4 included lactate level at 48 hours.

$\mathrm{OR}$, odds ratio; $\mathrm{Cl}$, confidence interval; $\mathrm{NA}$, not available.

${ }^{*} \mathrm{P}<0.05$. 
Odds ratio with $95 \% \mathrm{Cl}$

for poor neurologic outcome

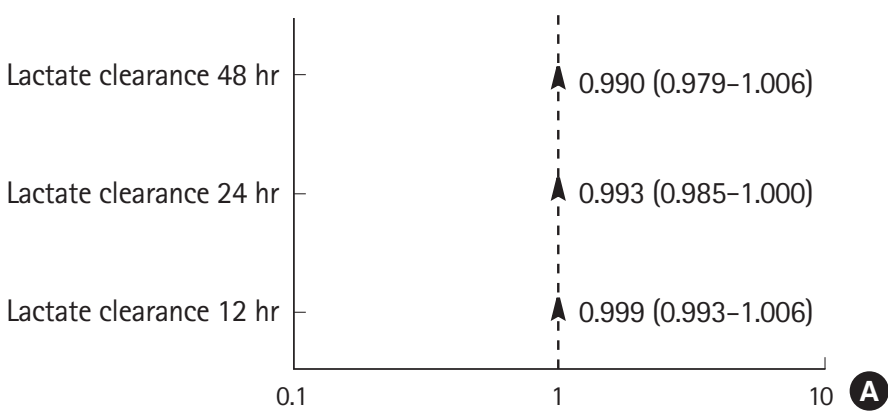

Odds ratio with $95 \% \mathrm{Cl}$

for in-hospital mortality

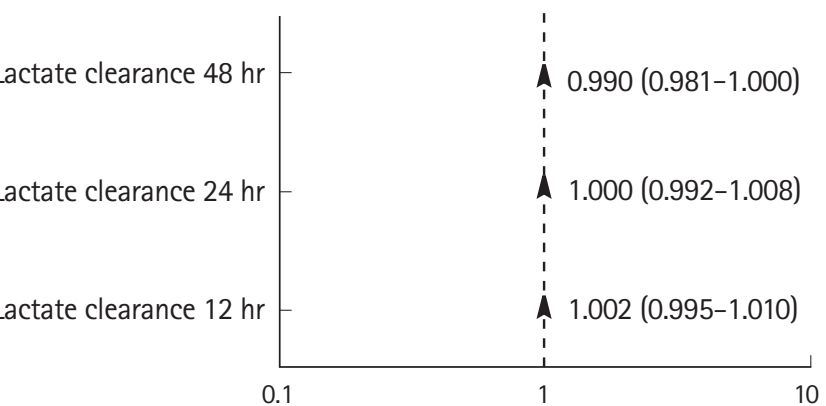

Fig. 4. Multivariate logistic regression model showing the association between lactate clearance and outcomes. (A) Lactate clearance at 12, 24, and 48 hours after admission was not associated with neurologic outcome. (B) Lactate clearance at 12, 24, and 48 hours after admission was not associated with in-hospital mortality. $\mathrm{Cl}$, confidence interval.

Table 4. Multivariate logistic regression analysis of characteristics associated with in-hospital mortality

\begin{tabular}{|c|c|c|c|c|}
\hline \multirow{2}{*}{ Characteristics } & \multicolumn{4}{|c|}{ OR $(95 \% \mathrm{Cl})$} \\
\hline & Model 1 & Model 2 & Model 3 & Model 4 \\
\hline Shockable rhythm & $0.400(0.192-0.833)^{*}$ & $0.400(0.192-0.833)^{*}$ & $0.400(0.192-0.833)^{*}$ & $0.378(0.171-0.835)^{*}$ \\
\hline SOFA & $1.237(1.133-1.352)^{*}$ & $1.237(1.133-1.352)^{*}$ & $1.237(1.133-1.352)^{*}$ & $1.114(1.006-1.234)^{*}$ \\
\hline \multicolumn{5}{|c|}{ Lactate levels (mmol/L) } \\
\hline Initial & $1.063(0.981-1.152)$ & NA & NA & NA \\
\hline At 12 hours & NA & $1.031(0.928-1.146)$ & NA & NA \\
\hline At 24 hours & NA & NA & $1.024(0.940-1.116)$ & NA \\
\hline At 48 hours & NA & NA & NA & $1.459(1.181-1.803)^{*}$ \\
\hline
\end{tabular}

Serum lactate levels at the different time points were separated into different multivariate models. Model 1 included initial lactate level, model 2 included lactate level at 12 hours after admission, model 3 included lactate level at 24 hours, and model 4 included lactate level at 48 hours.

$\mathrm{OR}$, odds ratio; $\mathrm{Cl}$, confidence interval; SOFA, sequential organ failure assessment; $\mathrm{NA}$, not available.

${ }^{*} \mathrm{P}<0.05$.

and poor neurologic outcome groups (Fig. 3). Serum lactate levels also differed between survivors and non-survivors and decreased over time $(\mathrm{P}<0.001)$, with no interaction between in-hospital mortality and time $(P=0.444)$ (Fig. 2). Post-hoc analysis showed that serum lactate levels at 48 hours after admission differed between survivors and non-survivors (Fig. 2). Lactate clearance at 48 hours after admission was different between survivors and non-survivors (Fig. 3).

\section{Association between lactate levels or clearance and out- comes}

Multivariate analysis revealed that an older age, non-shockable rhythm, non-cardiac etiology, longer downtime, and a lower GCS score were associated with a poor neurologic outcome (Table 3). Serum lactate levels at various time points were separated into different multivariate models. Higher serum lactate levels at 12 hours (odds ratio [OR], 1.157; 95\% confidence interval [Cl], 1.006 to 1.331$), 24$ hours $(\mathrm{OR}, 1.320 ; 95 \% \mathrm{Cl}, 1.084$ to 1.607$)$, and 48 hours $(\mathrm{OR}, 2.474 ; 95 \% \mathrm{Cl}, 1.459$ to 4.195$)$ after admission were associated with a poor neurologic outcome, whereas lactate level on admission $(\mathrm{OR}, 1.049 ; 95 \% \mathrm{Cl}, 0.962$ to 1.143$)$ was not associated with neurologic outcome (Table 3). Furthermore, lactate clearance was not associated with neurologic outcome at any time point (Fig. 4). Multivariate analysis revealed that a nonshockable rhythm and the SOFA score were associated with inhospital mortality (Table 4). A higher lactate level at 48 hours ( $O R$, 1.459; 95\% $\mathrm{Cl}, 1.181$ to 1.803 ) after admission was associated with an increased in-hospital mortality, whereas the lactate level on admission ( $\mathrm{OR}, 1.063 ; 95 \% \mathrm{Cl}, 0.981$ to 1.152$)$ and the lactate levels at 12 hours $(\mathrm{OR}, 1.031 ; 95 \% \mathrm{Cl}, 0.928$ to 1.146$)$ and 24 hours ( $O R, 1.024 ; 95 \% \mathrm{Cl}, 0.940$ to 1.116$)$ after admission were not associated with in-hospital mortality (Table 4). Additionally, lactate clearance was not associated with in-hospital mortality at any time point (Fig. 4).

\section{DISCUSSION}

In this retrospective cohort of cardiac arrest survivors treated 
with $\Pi \mathrm{M}$, we found that serum lactate levels at 12,24 , and 48 hours after admission were higher in patients with a poor neurologic outcome. Furthermore, serum lactate levels remained a surrogate marker even after adjusting for potential confounders. Lactate clearance at 12, 24, and 48 hours after admission was lower in patients with a poor neurologic outcome. However, lactate clearance was not a robust surrogate marker of neurologic outcome at any time point. A higher serum lactate level at 48 hours after admission was associated with increased in-hospital mortality. Lactate clearance at 48 hours after admission was lower in non-survivors; however, it did not remain a surrogate marker of in-hospital mortality following adjustment for confounders.

Previous studies have demonstrated the association between serum lactate levels and outcome in cardiac arrest survivors. ${ }^{7,9-14}$ Shinozaki et al. ${ }^{9}$ suggested that the serum lactate level was an independent predictor of neurologic outcome in cardiac arrest survivors. However, only $20.4 \%$ of the cohort in the study by Shinozaki et al. ${ }^{9}$ was treated with therapeutic hypothermia. Induced systemic hypothermia decreased microcirculation and peripheral tissue perfusion without impairment of systemic blood flow in out-of-hospital cardiac arrest survivors. ${ }^{18}$ Microvascular density and flow after cardiac arrest were influenced by body temperature, but not arterial pressure, cardiac output, or disease severity. ${ }^{19}$ Lactic acidosis can develop as a result of microcirculatory impairment when the body temperature is lowered. ${ }^{20}$ TM has been used as a standard treatment for comatose cardiac arrest survivors. ${ }^{5,6}$ All cardiac arrest survivors in our cohort were treated with TM. Therefore, the association between serum lactate levels and outcomes in cohorts treated with TTM should be reevaluated. Starodub et al. ${ }^{14}$ evaluated the use of the serum lactate level as a surrogate marker in a cohort of 199 cardiac arrest survivors treated with therapeutic hypothermia. They found that lower serum lactate levels at 12 and 24 hours, but not on admission, were associated with survival to discharge. Similarly, we found that the serum lactate levels at all time points up to 48 hours after admission, but not on admission, were robust prognostic indicators of neurologic outcome in our cohort. Lee et al. ${ }^{11}$ reported that there was no difference in initial lactate levels between patients with good and poor neurologic outcomes. Donnino et al. ${ }^{12}$ also demonstrated that the initial lactate level did not remain a surrogate maker of survival or neurologic outcome after adjusting for potential confounders in a cohort of 100 cardiac arrest survivors treated with TTM. The serum lactate level during post-resuscitation care is affected by multiple factors, including the post-cardiac arrest systemic inflammatory response, myocardial stunning, and secondary ischemic insults such as seizure, infection, and sepsis, whereas the initial serum lactate level depends predominantly on ischemic insult during downtime and hypoperfusion before collapse. ${ }^{21-24}$ Therefore, the serum lactate level on admission cannot be a robust surrogate marker of survival or neurologic outcome in cardiac arrest survivors receiving postresuscitation care. Notably, several earlier studies did not measure serum lactate levels beyond 24 hours after cardiac arrest. ${ }^{9,10,12,14}$ Hence, they could not demonstrate the association between serum lactate levels beyond 24 hours after cardiac arrest and outcomes. However, we found an independent association between serum lactate levels at 48 hours and neurologic outcome or in-hospital mortality.

The serum lactate level significantly decreased over time in the present study. The change in serum lactate level is related to lactate clearance. Effective lactate clearance was reported as another surrogate marker in cardiac arrest survivors. ${ }^{10-13}$ Donnino et al. ${ }^{10}$ first demonstrated that a greater lactate clearance at 12 hours was associated with reduced mortality in a cohort of 79 cardiac arrest survivors who did not receive TTM. The association between lactate clearance and clinical outcome should be reevaluated considering the effect of temperature on serum lactate levels. Lee et al. ${ }^{11}$ showed that a greater lactate clearance at 6 and 12 hours was a robust prognostic indicator of a good neurologic outcome in out-of-hospital cardiac arrest survivors treated with therapeutic hypothermia. Donnino et al. ${ }^{12}$ demonstrated that a greater lactate clearance at 12 hours was associated with good neurologic outcome and survival in a cohort of cardiac arrest survivors treated with TMM, whereas the lactate clearance at 24 hours was not associated with outcomes. The serum lactate level precipitously decreased within 6 hours after admission and then decreased slowly, as reported by previous studies. ${ }^{10-14}$ Therefore, the difference in early lactate clearance rather than late lactate clearance may be more prominent, and early lactate clearance may be a robust marker. In contrast to previous studies, we found that lactate clearance had no association with neurologic outcome or in-hospital mortality after adjusting for potential confounders. Lactate clearance increased over time in survivors and patients with a good neurologic outcome, whereas it plateaued at $30 \%$ to $40 \%$ in non-survivors and patients with a poor neurologic outcome in previous studies. ${ }^{10,12}$ However, in the present study, lactate clearance increased over time in patients with a poor neurologic outcome and in non-survivors, which was similar to that for patients with a good neurologic outcome and survivors. This suggested that perfusion during post-resuscitation care was well managed even in patients with a poor neurologic outcome and in non-survivors. However, perfusion needs to be considered with respect to whether or not a vasopressor was needed. Cocchi et al. ${ }^{25}$ demonstrated that lactate levels, together 
with the need for a vasopressor, was an accurate predictor of mortality in out-of-hospital cardiac arrest survivors. Unfortunately, information regarding the need for vasopressors was not available in our data set. Future studies should be performed taking into consideration the requirement for a vasopressor.

Nevertheless, the serum lactate levels at all time points in the present study were similar to those reported in the study by Lee et al. ${ }^{11}$ although, in contrast, lactate clearance was not associated with neurologic outcome or in-hospital mortality at any time point in the present study. This difference can be attributed to differences in the baseline characteristics. The cohort in the study by Lee et al." ${ }^{11}$ was younger and showed a higher rate of shockable rhythm. The cohort in the study by Donnino et al. ${ }^{12}$ showed a higher rate of shockable rhythm and a shorter downtime. Age, shockable rhythm, and downtime were independent prognostic indicators in the present study. These differences in baseline characteristics may explain the lack of an association between lactate clearance and clinical outcomes in the present study.

There are several limitations of the present study. First, although measurement of serum lactate levels formed part of the protocol, some lactate values were missing. Thirty (10.6\%) of 312 patients were excluded because of missing data, and 35 patients were excluded because death occurred during TTM. This may have led to selection bias. However, in most cases, death during TTM occurred before achieving the target temperature, due to a circulatory shock. It was decided to exclude patients who died during $\Pi M$, as death during $T \mathrm{M}$ did not allow completion of TTM and circulatory shock resulted in extremely high serum lactate levels, which might have affected the results. Second, data were collected prospectively, but this was a retrospective cohort study performed at a single center using secondary data from electronic medical records, which could also have led to selection bias. Third, vasoactive agents can influence lactate production, but data concerning the use of vasoactive agents during post-resuscitation care were not available. Calculating the cumulative dose of vasoactive agents or dose at the time serum lactate levels are obtained is challenging. Future studies are required to consider the need for vasoactive agents. Fourth, despite our efforts, there may have been potential confounding factors that affected hemodynamics and lactate levels. Fifth, we excluded patients who died during TM. Non-survivors died mainly as a result of post-cardiac arrest shock within 3 days after ROSC and had higher serum lactate levels. ${ }^{26}$ The finding of no difference in initial serum lactate levels in the present study might have been influenced by the exclusion of patients who died during TM.

In conclusion, lactate clearance was not a robust surrogate marker of neurologic outcome or in-hospital mortality at any time point. However, lactate levels at 12, 24, and 48 hours after admission were associated with neurologic outcome at discharge in cardiac arrest survivors treated with TTM. The lactate level at 48 hours after admission was associated with survival to discharge.

\section{CONFLICT OF INTEREST}

No potential conflict of interest relevant to this article was reported.

\section{REFERENCES}

1. Hypothermia after Cardiac Arrest Study Group. Mild therapeutic hypothermia to improve the neurologic outcome after cardiac arrest. N Engl J Med 2002;346:549-56.

2. Bernard SA, Gray TW, Buist MD, et al. Treatment of comatose survivors of out-of-hospital cardiac arrest with induced hypothermia. N Engl J Med 2002;346:557-63.

3. Sunde $K$, Pytte $M$, Jacobsen $D$, et al. Implementation of a standardised treatment protocol for post resuscitation care after out-of-hospital cardiac arrest. Resuscitation 2007;73: 29-39.

4. Gaieski DF, Band RA, Abella BS, et al. Early goal-directed hemodynamic optimization combined with therapeutic hypothermia in comatose survivors of out-of-hospital cardiac arrest. Resuscitation 2009;80:418-24.

5. Callaway CW, Donnino MW, Fink EL, et al. Part 8: post-cardiac arrest care. 2015 American Heart Association Guidelines Update for Cardiopulmonary Resuscitation and Emergency Cardiovascular Care. Circulation 2015;132(18 Suppl 2):S465-82.

6. Nolan JP, Soar J, Cariou A, et al. European Resuscitation Council and European Society of Intensive Care Medicine Guidelines for Post-resuscitation Care 2015: Section 5 of the European Resuscitation Council Guidelines for Resuscitation 2015. Resuscitation 2015;95:202-22.

7. Mikkelsen ME, Miltiades AN, Gaieski DF, et al. Serum lactate is associated with mortality in severe sepsis independent of organ failure and shock. Crit Care Med 2009;37:1670-7.

8. Kliegel A, Losert $H$, Sterz F, et al. Serial lactate determinations for prediction of outcome after cardiac arrest. Medicine (Baltimore) 2004;83:274-9.

9. Shinozaki K, Oda S, Sadahiro T, et al. Blood ammonia and lactate levels on hospital arrival as a predictive biomarker in patients with out-of-hospital cardiac arrest. Resuscitation 2011; 82:404-9.

10. Donnino MW, Miller J, Goyal N, et al. Effective lactate clear- 
ance is associated with improved outcome in post-cardiac arrest patients. Resuscitation 2007;75:229-34.

11. Lee TR, Kang MJ, Cha WC, et al. Better lactate clearance associated with good neurologic outcome in survivors who treated with therapeutic hypothermia after out-of-hospital cardiac arrest. Crit Care 2013;17:R260.

12. Donnino MW, Andersen LW, Giberson T, et al. Initial lactate and lactate change in post-cardiac arrest: a multicenter validation study. Crit Care Med 2014;42:1804-11.

13. Williams TA, Martin R, Celenza $A$, et al. Use of serum lactate levels to predict survival for patients with out-of-hospital cardiac arrest: a cohort study. Emerg Med Australas 2016;28: 171-8.

14. Starodub R, Abella BS, Grossestreuer AV, et al. Association of serum lactate and survival outcomes in patients undergoing therapeutic hypothermia after cardiac arrest. Resuscitation 2013;84:1078-82.

15. Peberdy MA, Callaway CW, Neumar RW, et al. Part 9: postcardiac arrest care. 2010 American Heart Association Guidelines for Cardiopulmonary Resuscitation and Emergency Cardiovascular Care. Circulation 2010;122(18 Suppl 3):S768-86.

16. Vincent $J L$, de Mendonca $A$, Cantraine $F$, et al. Use of the SOFA score to assess the incidence of organ dysfunction/failure in intensive care units: results of a multicenter, prospective study. Working group on "sepsis-related problems" of the European Society of Intensive Care Medicine. Crit Care Med 1998;26:1793-800.

17. Booth CM, Boone RH, Tomlinson G, Detsky AS. Is this patient dead, vegetative, or severely neurologically impaired? Assessing outcome for comatose survivors of cardiac arrest. JAMA 2004;291:870-9.

18. van Genderen ME, Lima A, Akkerhuis M, Bakker J, van Bom- mel J. Persistent peripheral and microcirculatory perfusion alterations after out-of-hospital cardiac arrest are associated with poor survival. Crit Care Med 2012;40:2287-94.

19. Donadello K, Favory R, Salgado-Ribeiro D, et al. Sublingual and muscular microcirculatory alterations after cardiac arrest: a pilot study. Resuscitation 2011;82:690-5.

20. Buijs EA, Verboom EM, Top AP, et al. Early microcirculatory impairment during therapeutic hypothermia is associated with poor outcome in post-cardiac arrest children: a prospective observational cohort study. Resuscitation 2014;85:397404.

21. Adrie $C$, Adib-Conquy M, Laurent I, et al. Successful cardiopulmonary resuscitation after cardiac arrest as a "sepsis-like" syndrome. Circulation 2002;106:562-8.

22. Chalkias $A$, Xanthos T. Pathophysiology and pathogenesis of post-resuscitation myocardial stunning. Heart Fail Rev 2012; 17:117-28.

23. Mani $R$, Schmitt SE, Mazer M, Putt ME, Gaieski DF. The frequency and timing of epileptiform activity on continuous electroencephalogram in comatose post-cardiac arrest syndrome patients treated with therapeutic hypothermia. Resuscitation 2012;83:840-7.

24. Mongardon N, Perbet $\mathrm{S}$, Lemiale $\mathrm{V}$, et al. Infectious complications in out-of-hospital cardiac arrest patients in the therapeutic hypothermia era. Crit Care Med 2011;39:1359-64.

25. Cocchi MN, Miller J, Hunziker S, et al. The association of lactate and vasopressor need for mortality prediction in survivors of cardiac arrest. Minerva Anestesiol 2011;77:1063-71.

26. Lemiale $V_{\text {, Dumas }} F_{1}$ Mongardon $N$, et al. Intensive care unit mortality after cardiac arrest: the relative contribution of shock and brain injury in a large cohort. Intensive Care Med 2013;39:1972-80. 